University of Nebraska - Lincoln

DigitalCommons@University of Nebraska - Lincoln

USDA Wildlife Services - Staff Publications

U.S. Department of Agriculture: Animal and Plant Health Inspection Service

2021

\title{
Variation in reversal learning by three generalist mesocarnivores
}

Lauren A. Stanton

University of Wyoming, laurenashleystanton@gmail.com

Eli S. Bridge

University of Oklahoma

Joost Huizinga

OpenAl, San Francisco, CA

Shylo R. Johnson

USDA/APHIS/WS National Wildlife Research Center

Julie K. Young

USDA/APHIS/WS National Wildlife Research Center

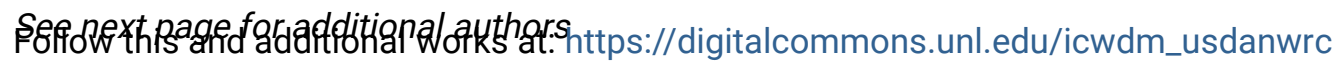

Part of the Natural Resources and Conservation Commons, Natural Resources Management and

Policy Commons, Other Environmental Sciences Commons, Other Veterinary Medicine Commons,

Population Biology Commons, Terrestrial and Aquatic Ecology Commons, Veterinary Infectious Diseases

Commons, Veterinary Microbiology and Immunobiology Commons, Veterinary Preventive Medicine,

Epidemiology, and Public Health Commons, and the Zoology Commons

Stanton, Lauren A.; Bridge, Eli S.; Huizinga, Joost; Johnson, Shylo R.; Young, Julie K.; and Benson-Amram, Sarah, "Variation in reversal learning by three generalist mesocarnivores" (2021). USDA Wildlife Services Staff Publications. 2453.

https://digitalcommons.unl.edu/icwdm_usdanwrc/2453

This Article is brought to you for free and open access by the U.S. Department of Agriculture: Animal and Plant Health Inspection Service at DigitalCommons@University of Nebraska - Lincoln. It has been accepted for inclusion in USDA Wildlife Services - Staff Publications by an authorized administrator of DigitalCommons@University of Nebraska - Lincoln. 


\section{Authors}

Lauren A. Stanton, Eli S. Bridge, Joost Huizinga, Shylo R. Johnson, Julie K. Young, and Sarah Benson-Amram 


\title{
Variation in reversal learning by three generalist mesocarnivores
}

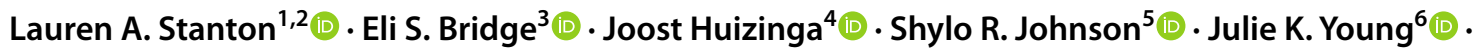 \\ Sarah Benson-Amram ${ }^{1,2}$ (B)
}

Received: 2 March 2020 / Revised: 25 September 2020 / Accepted: 29 September 2020 / Published online: 24 November 2020

(c) Springer-Verlag GmbH Germany, part of Springer Nature 2020

\begin{abstract}
Urbanization imposes novel challenges for wildlife, but also provides new opportunities for exploitation. Generalist species are commonly found in urban habitats, but the cognitive mechanisms facilitating their successful behavioral adaptations and exploitations are largely under-investigated. Cognitive flexibility is thought to enable generalists to be more plastic in their behavior, thereby increasing their adaptability to a variety of environments, including urban habitats. Yet direct measures of cognitive flexibility across urban wildlife are lacking. We used a classic reversal-learning paradigm to investigate the cognitive flexibility of three generalist mesocarnivores commonly found in urban habitats: striped skunks (Mephitis mephitis), raccoons (Procyon lotor), and coyotes (Canis latrans). We developed an automated device and testing protocol that allowed us to administer tests of reversal learning in captivity without extensive training or experimenter involvement. Although most subjects were able to rapidly form and reverse learned associations, we found moderate variation in performance and behavior during trials. Most notably, we observed heightened neophobia and a lack of habituation expressed by coyotes. We discuss the implications of such differences among generalists with regard to urban adaptation and we identify goals for future research. This study is an important step in investigating the relationships between cognition, generalism, and urban adaptation.
\end{abstract}

Keywords Behavioral adaptation $\cdot$ Comparative cognition $\cdot$ Carnivore $\cdot$ Flexibility $\cdot$ Generalism $\cdot$ Urbanization

Electronic supplementary material The online version of this article (https://doi.org/10.1007/s10071-020-01438-4) contains supplementary material, which is available to authorized users.

Lauren A. Stanton

laurenashleystanton@gmail.com

1 Department of Zoology and Physiology, University of Wyoming, Laramie, WY, USA

2 Program in Ecology, University of Wyoming, Laramie, WY, USA

3 Oklahoma Biological Survey, University of Oklahoma, Norman, OK, USA

4 OpenAI, San Francisco, CA, USA

5 USDA/APHIS/WS National Wildlife Research Center, Fort Collins, CO, USA

6 USDA/APHIS/WS National Wildlife Research Center - Predator Research Facility, Millville, UT, USA

\section{Introduction}

Urbanization is rapidly changing the planet and has been linked to extinctions and evolutionary change in organisms across the globe (Pimm et al. 2014; Alberti et al. 2016). Given the projected biodiversity loss stemming from urbanization (Seto et al. 2012), understanding the mechanisms that affect a species' propensity to avoid or adapt to urban habitats remains a priority for conservation and urban planning (Johnson and Munshi-South 2017). Urban habitats are typically characterized by the same broad environmental transformations: an increase in humans and artificial entities (e.g., lights, acoustics, substrates, vehicles, trash, chemicals) and a decrease in natural resources and landscape connectivity (Grimm et al. 2008; Sih et al. 2011). The anthropogenic disturbances that occur in urban habitats impose new challenges for wildlife, but anthropogenic resources (e.g., food, shelter) also provide new opportunities for exploitation (Lowry et al. 2013). Consequently, some species not only persist but actually thrive in urbanized habitats (McKinney 2006). These successful species are usually found to be 
ecological generalists, characterized by their ability to use a diversity of food and habitat types (Devictor et al. 2008; Ducatez et al. 2018). Yet the mechanisms underlying the adaptive, exploitative skills of generalists represents a critical gap in our knowledge (Overington et al. 2011).

Ecological generalism may have coevolved with a suite of cognitive abilities that allow generalists to cope with environmental variability (Lefebvre et al. 1997; Sol 2009; Overington et al. 2011; Ducatez et al. 2015; Sol et al. 2016; Navarrete et al. 2016). The cognitive buffer hypothesis suggests that advanced cognitive abilities associated with large relative brain size, such as rapid learning, innovation, and flexibility, allow animals to modify their behavior in adaptive ways when confronted with challenges in their environment (Sol 2009). In accordance with the cognitive buffer hypothesis, generalists are exposed to diverse environmental conditions that require a capacity for rapid, flexible responses to new or local cues (Mettke-Hofmann 2014; Sol et al. 2016). Such a capacity includes the exploitation of various habitats, foods, and shelters while avoiding the novel dangers associated with such transitions, such as the consumption of noxious foods or increased risk of predation (Greenberg 2003; Sol 2003; Robertson et al. 2013). Cognitive flexibility may, therefore, allow generalists to be plastic in their behavior, which increases their adaptability (Godfrey-Smith 1996; Sol and Lefebvre 2000; Sol et al. 2002, 2013; Wright et al. 2010; Mettke-Hofmann 2014) and serves as a potential mechanism for urban adaptation (Maklakov et al. 2011; Sih et al. 2011; Snell-Rood and Wick 2013; Sol et al. 2013). For example, both brain size and dietary innovation rate have been implicated in the success of many widespread species (Lefebvre et al. 1997; Sol and Lefebvre 2000; Sol et al. 2002, 2008; Maklakov et al. 2011; Overington et al. 2011; SnellRood and Wick 2013; Ducatez et al. 2015). Nevertheless, establishing the link between the cognitive traits of generalists and their successful adaptation to urbanization requires further empirical support.

The cognitive flexibility of an animal is commonly assessed using a test known as reversal learning, which was originally designed as an assessment of animal intelligence (Mackintosh et al. 1968). In this paradigm, previously learned reward associations are reversed and the ability of the animal to respond to the new contingency by changing its behavior remains a widely accepted measure of flexibility (Audet and Lefebvre 2017; Izquierdo et al. 2017). Reversal learning is thought to involve two different aspects of learning: an initial, more basic associative learning of a stimulus and a response (i.e., acquisition phase), and a second, more flexible reversed learning ability that requires additional executive control (i.e., reversal phase) (Lai et al. 1995; Chow et al. 2015; Buechel et al. 2018; Madden et al. 2018). Specifically, when an animal is presented with the challenge of a reversal, it must first inhibit its inclination to select the previously correct response and then shift its attention to the new stimuli (Shettleworth 2010). Although most (if not all) animals can be expected to learn simple associations (Morand-Ferron 2017), less flexible individuals are expected to demonstrate difficulty responding to a reversed association and, therefore, make a high number of errors during the onset of a reversal event. In contrast, highly flexible individuals experience less difficulty in altering their behavior and, therefore, make fewer errors. As an animal learns the affordances of this task, it is also expected that they will make fewer errors across reversals and perhaps even demonstrate an optimal, more advanced learning strategy, wherein only one error is made per reversal (i.e., win stay-lose shift strategy; Mackintosh et al. 1968; Macphail and Bolhuis 2001; Shettleworth 2010). Thus, the cognitive flexibility of an animal can be measured by the onset of a single reversal event or across serial reversals; tests that may emulate challenges found in complex or changing environments, such as switching foraging strategies (Tebbich and Teschke 2014), resource tracking (Hermer et al. 2018), or maintaining social competency (Bond et al. 2007). Indeed, performance in reversal learning has been linked to many cognitive and ecological traits of interest, including brain size (Buechel et al. 2018), social complexity (Bond et al. 2007; Ashton et al. 2018), and habitat unpredictability (Tebbich and Teschke 2014; but see Croston et al. 2017; Hermer et al. 2018). Furthermore, the neural basis of reversal learning has been well-established in the lab (Izquierdo et al. 2017). Since reversal learning is a validated measure of the cognitive underpinnings of behavioral plasticity and parallels ecologically relevant challenges that animals face in novel, complex, or changing environments, it can serve as a tool to investigate the link between the cognition of generalists and urban adaptation.

Mammals comprise a rich and underutilized taxon for investigations of cognition and behavioral adaptation to urbanization. The evolution of intelligence in mammalian carnivores (i.e., mammals within the order Carnivora) may have been driven by ecological complexity (Holekamp and Benson-Amram 2017), and behavioral plasticity has been suggested as important for urban carnivores (Bateman and Fleming 2012; Barrett et al. 2019; Murray and St. Clair 2015; Young et al. 2019a). Large and small mammalian carnivores are underrepresented in urban areas (Bateman and Fleming 2012), and large carnivores are typically the first to disappear as a result of an increased human presence (McKinney 2002). In contrast, several medium-sized, generalist carnivores (i.e., "mesocarnivores") in North America, such as striped skunks (Mephitis mephitis; henceforth "skunks"), raccoons (Procyon lotor), and coyotes (Canis latrans) are successful at persisting despite often being targeted by humans as "nuisance species" (Gehrt 2004; Bateman and Fleming 2012; Barrett et al. 2019). Thus, these 
species may offer new insight on the relationships between cognition, generalism and urban adaptation. Indeed, early comparative cognition research on mesocarnivores, including skunks and raccoons, suggests that these species are capable of discrimination and reversal learning (Fields 1936; Shell and Riopelle 1957; Johnson and Michels 1958; Warren and Warren 1962; Gossette et al. 1968; Doty and Combs 1969). However, these studies were conducted in a laboratory setting with heavy experimenter involvement and may have limited applicability to wild animals. Although fewer studies of traditional discrimination and reversal learning have been conducted with coyotes, captive coyotes have proven capable of discriminating between different quantities of food (Baker et al. 2011; but see Mahamane et al. 2014). Captive coyotes also have demonstrated skill in matching their behavior to changing reward contingencies, which is suggestive of behavioral plasticity (Gilbert-Norton et al. 2009). Despite the tenacity of urban mesocarnivores, the challenges of working with such species, especially in the wild, has hindered investigation of their cognitive flexibility (e.g., Pettit 2010; Stanton et al. 2017) and thus remains generally under-investigated in comparison to other taxa (e.g., birds; but see Gossette et al. 1968; Gilbert-Norton et al. 2009; Daniels et al. 2019).

To generate new insights on mesocarnivore cognition and thereby address critical gaps in knowledge regarding the link between the cognition of generalists and urban adaptation, we developed an automated device and protocol to directly measure the cognitive flexibility of skunks, raccoons, and coyotes in captivity using a classic reversal-learning paradigm. Although these mesocarnivores differ in many traits, including brain morphology, sociality, reproduction, and foraging strategies, all three species are considered to be predominately nocturnal, dietary and habitat generalists that are commonly found in cities in North America (Gehrt 2004; Gehrt et al. 2010; Jardim-Messeder et al. 2017; Stankowich and Romero 2017). Given that behavioral plasticity and ecological generalism may have coevolved, we expect that skunks, raccoons, and coyotes are cognitively flexible, and that this aids in their exploitation of urban habitats. Therefore, we predicted that all three species would form rapid associations and demonstrate cognitive flexibility as evidenced by a low number of errors made during the reversal of a learned behavior. Our methodology allowed us to assess the reversal-learning ability of mesocarnivores without any hands-on training, which has the potential for field applications. Although similar methodologies have been developed for reversal learning in studies of wild birds (MorandFerron, Hamblin, et al. 2015a, b; Cauchoix et al. 2017; Tello-Ramos et al. 2018; Bridge et al. 2019), to the best of our knowledge this methodology has yet to be applied to mammalian carnivores. Thus, our study extends the body of work on automated cognition testing to an understudied yet remarkably successful guild, thereby advancing our ability to investigate the connections between generalism, cognition, and urban adaptation.

\section{Methods}

\section{Study sites and general procedure}

Trials were conducted at two USDA National Wildlife Research Center (NWRC) facilities and this study was approved by NWRC Institute for Animal Care and Use Committee (QA-2825). Skunks $(n=4)$ and raccoons $(n=11)$ were tested at the NWRC headquarters in Fort Collins, CO, whereas coyotes $(n=6)$ were tested at the NWRC's Predator Research Facility in Millville, UT (see Electronic Supplementary Material (ESM) for husbandry and housing information). Animal subjects were selected on their likelihood of voluntary participation (i.e., exhibited low levels of fear and high levels of food motivation; see ESM). Trials were administered by an automated device resembling a classic operant conditioning chamber or "Skinner Box" (Skinner 1938) using a two-choice, spatial paradigm. The device featured two round buttons (placed at an angle for skunks and placed vertically for raccoons) or rectangular foot pedals (placed on the ground for coyotes) on either side of a food dispensing chute (Fig. 1). Each button was associated with an LED light to indicate the device was on and active. Subjects were required to press one of the two buttons, either on the left or right side, to receive a food reward. When the subject pressed the correct button (i.e., positive stimulus), the device automatically released a small food reward. When the subject pressed the incorrect button (i.e., negative stimulus), the device initiated a brief $10 \mathrm{~s}$ time-out period, where the LED lights shut off and the device became unresponsive. Trials were delivered in blocks of 10 , and subjects were required to meet a $90 \%$ learning criterion (i.e., select the correct button in 9 out of 10 consecutive trials) before a block was considered passed (Cauchoix et al. 2017). If the subject did not meet the $90 \%$ criterion, the block was considered a failure, and a new block of 10 trials began.

We considered the first test delivered every night, in which the subject had to make an initial discrimination between the two stimuli and meet the $90 \%$ criterion, to be a "pre-reversal block". The initial, correct stimulus (i.e., right vs. left button) in a subject's first pre-reversal block was randomly assigned. The rewarded stimulus in subsequent pre-reversal blocks was alternated across all testing sessions for a subject. Tests following the prereversal block within a single testing session were considered to be reversals. Whenever a block was passed, a reversal event was initiated by the device, whereby the current reward association was reversed (i.e., the negative 
Fig. 1 Images of the devices used to deliver trials to $\mathbf{a}$ skunks, $\mathbf{b}$ raccoons, and $\mathbf{c}$ coyotes. Buttons were placed in different positions so that they were easily accessible to each species, and the device used with coyotes was smaller than that used with skunks and raccoons
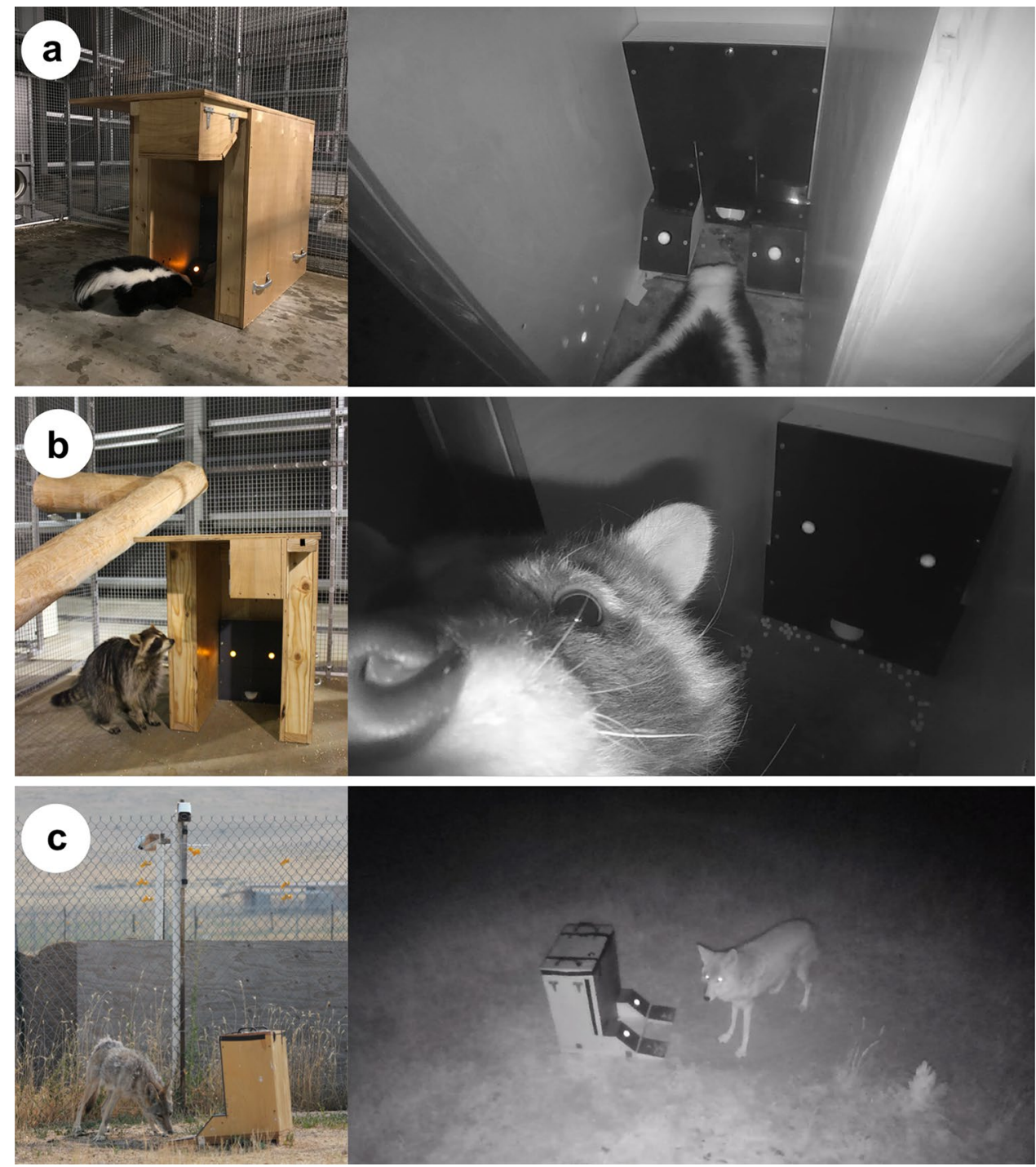

stimulus became the positive stimulus and vice-versa) and a new block of trials began. Subjects were not limited in the number of the reversals they could achieve in a single testing session, and our goal was to obtain a minimum of 30 reversals per individual over the course of several testing sessions so that performance could be assessed across time. Subjects were tested approximately once every $24 \mathrm{~h}$ for the duration of their trials (median $=24.17 \mathrm{~h}$, range $=17.89-337.02 \mathrm{~h}$ ), although this was subject to animal and researcher availability (see ESM). If a subject did not complete a pre-reversal or a reversal prior to the end of its testing session, this incomplete block was abandoned and the subject started a new pre-reversal block in its subsequent testing session. All trials were automated and filmed so that an experimenter was not present nor actively involved in trials (video footage from the skunk, raccoon, and coyote trials can be found in ESM Videos
1,2 , and 3 , respectively). The device kept a record of all trials administered.

\section{Skunk and raccoon protocol}

Skunks (4 males, 0 females) were all captive-bred subjects and raccoons (6 males, 5 females) were all wild-caught subjects (see ESM). Subjects were not food-deprived for this experiment and always had access to their food and water bowls during trials. They were tested individually, at night, and the testing device was placed in a subject's home enclosure at the beginning of a testing session and removed at the end of the session. The device used with skunks and raccoons was equipped with an infrared (night vision) camera and a break-beam sensor (Fig. 1a, b). The break-beam sensor was able to detect the presence of an animal inside of the device. We used this feature to both habituate subjects to 
the device in their initial testing sessions by providing free rewards for simply entering the device, and to signal the start of trials upon their entry into the device for each subsequent testing session (see ESM).

The reward used for skunks and raccoons was a mix of cereal and dog kibble that differed from their normal diet (see ESM). To train the animals to target the buttons and discourage the development of a side bias, both right and left buttons were initially baited with a food cue during the subject's first testing session (see ESM). During this time, subjects could press either button and receive a reward for a maximum of 10 pushes before the first pre-reversal test began. Testing sessions initially lasted up to $300 \mathrm{~min}$ to allow for habituation but were reduced to $30-90 \mathrm{~min}$ after subjects had begun to engage in testing (see ESM). Skunks and raccoons received one testing session per night for a maximum of 14 nights or a minimum of 30 reversals achieved (whichever occurred first). However, if a subject never approached the device within its first five testing sessions or did not receive their free rewards within eight testing sessions, the subject was considered uninterested and was removed from further testing.

\section{Coyote protocol}

Adult coyotes ( 5 males, 1 female) comprised a mix of wildcaught $(n=2)$ and captive-born $(n=4)$ individuals, but all received identical care after reaching 15 weeks of age (see ESM). Because coyotes have demonstrated neophobia toward large, novel objects (Mettler and Shivik 2007; Windberg 2008), several adjustments were made to our protocol and to the testing device upon the start of coyote trials. The device remained in the subject's enclosure full time for a minimum of 40 days, and testing sessions lasted for many hours, beginning at dusk and ending the following morning. Coyotes received a minimum of 25 testing sessions (i.e., when the device was active). The device was powered off while not in use, and a cover was placed over the interface so coyotes could not access the foot pedals outside of testing sessions. Unlike the devices used in the skunk and raccoon trials, the coyote device used was smaller, less like a chamber (i.e., no sides), and lacked a break-beam sensor and camera (Fig. 1c). Instead, the night vision camera used to film trials was mounted away from the device on the fencing of the animal's enclosure and remained present throughout the study. The reward was a mix of dog kibble and sausage, which differed from their normal diet (see ESM). For habituation, the device was programmed to automatically deliver food every $40 \mathrm{~min}$ throughout the initial testing sessions, regardless of where the coyote was in its enclosure. The foot pedals used for making a selection were constructed to lie flat on the ground and projected away from the device so that the coyotes could maintain some distance from the main part of the device during testing. Pedals were heavily baited every night with high-value food rewards (e.g., chicken liver, sausage, mink food), and coyotes were allowed to push on the baited pedals for an extended period of time (i.e., several testing sessions) before trials began. Thus, the habituation period was extended beyond that allocated to the skunks and raccoons, and coyotes received longer testing sessions in general. To further motivate participation, some of the coyotes were transitioned from their highly preferred, meatbased diet to a kibble diet, and some coyotes were also tested both alone and with their mate present (see ESM Table 1).

Although extended testing sessions were necessary, we were concerned that prolonged gaps in time (i.e., potentially several hours) between selections would result in memory loss and that this would inhibit our ability to make comparisons in performance among study subjects. Therefore, to emulate the testing experience of the skunks and raccoons, we initiated a block reset if the device went untouched by a coyote for more than $40 \mathrm{~min}$ (i.e., the maximum amount of time between selections observed by the skunks and raccoons). In other words, if the device detected no selections for $40 \mathrm{~min}$, any ongoing blocks were canceled, reset to 0 , and not included in analysis. Furthermore, we found that the $10 \mathrm{~s}$ time-out period upon an incorrect selection was too long for coyotes and would cause abandonment of testing. We, therefore, dropped the time-out period from 10 to $2 \mathrm{~s}$, which allowed us to better maintain coyote interest.

\section{Prior experience}

In the development of appropriate methods for this experiment, including the construction of the testing devices, some of the subjects were exposed to pilot versions of the testing device. This exposure was necessary for us to create a device and an automated protocol that was most suitable for testing mesocarnivores. Due to the time and resource constraints of our study, we decided to continue testing individuals that may have had initial, prior experience, because they were already habituated to our experimental setup. We expected that this prior experience might have affected individual performance in this task. Specifically, we estimated that animals having more prior experience would make fewer errors during testing than individuals that lacked any prior experience (Izquierdo et al. 2017). We, therefore, assigned a category to each subject based on the level of prior experience they had with the devices: group A were subjects that had experience with one or more pilot devices and completed a small number (1-13) of blocks (i.e., moderate experience; $n=5$ ), group B had experience with one or more pilot devices but did not complete blocks (i.e., low experience; $n=5$ ), and group $\mathrm{C}$ had no prior experience $(n=11)$. 


\section{Statistical analysis}

We tested (1) the ability of our subjects to improve their performance over time and (2) investigated factors that may have contributed to variation in performance. We measured performance by the number of errors an individual made before reaching criterion (i.e., before completing a reversal). All analyses were performed using Program R (R Core Team 2018). We first used Poisson regression to test how reversal number, individual ID, species, and prior experience (fixed effects) affected performance (i.e., number of errors made by subjects) using package lme4 (Bates et al. 2015). Although we intended to include additive models for all fixed effects, as well as additional traits such as sex and origin, we encountered data limitations that prevented us from building more complex models (see below). We evaluated model fit and parsimony using Akaike's Information Criterion corrected for small sample sizes (AICc) using package MuMIn (Bartoń 2018). Furthermore, we identified the amount of variation explained by each fixed effect by calculating $R^{2}$ values for each model. We also assessed whether duration (number of hours) between testing sessions could have affected participation and testing performance. Although we found a weak trend that suggested subjects may have completed more reversals after a prolonged break from testing, these results were non-significant (see ESM).

To evaluate variation in individual performance, we next built individual learning curves using Poisson regression to compare the number of errors each individual made during the first reversal ( $y$-intercept) and the rate at which the number of errors changed as reversal number increased (slope). We considered a subject to have improved in its performance if there was a decreasing trend in the number of errors made or the number of blocks an individual needed to meet learning criterion across reversals. Lastly, we tested each individual for a side bias, or the tendency for an individual to make more or fewer errors based on the side (left vs. right) assigned as the positive stimulus. To evaluate any side bias, we again used Poisson regression to model the effect of reversal number on the number of errors made while including the assigned positive stimulus (left or right) as a fixed effect.

To better understand the specific learning processes that subjects underwent in this experiment, we assessed the sequence of choices subjects made across testing sessions. As in other studies of reversal learning, we expected that subjects may develop an advanced, rule-based strategy to improve in performance, such as win stay-lose shift, or that subjects may experience a type of learning barrier that would inhibit improvement, such as proactive or retroactive interference (Anderson and Neeley 1996). Using analytical methods similar to those of recent reversal learning studies (Liu et al. 2016; Bridgeman and Tattersall 2019), we created pairs of successive choices starting at the beginning of a testing session and ending at the last completed block of that testing session for all subjects (i.e., included the pre-reversal block and subsequent reversal blocks for a testing session). We labeled each pair of choices as one of four possibilities: win stay (first choice correct, next choice correct), lose shift (first choice incorrect, next choice correct), win shift (first choice correct, next choice incorrect), and lose stay (first choice incorrect, next choice incorrect). We calculated the number of pairs for each category and divided it by all of the pairs made in a testing session, resulting in the proportion of each category per testing session. We expected that if subjects developed a win stay-lose shift strategy, we would see the proportion of win stay and lose shift responses increase, while the alternative, incorrect responses (i.e., win shift-lose stay) decreased. However, if subjects were experiencing a learning barrier, such as proactive or retroactive interference, or lacked inhibitory control, then we generally expected to see a high proportion of incorrect responses. We, therefore, combined the proportion of win stay responses with lose shift responses and win shift responses with lose stay responses and modeled the effect of testing session on the proportion of each strategy exhibited by each species, while controlling for individual variation by allowing the intercept to vary among individuals (package glmmTMB; Brooks et al. 2017).

\section{Results}

The majority of skunks ( 3 of 4 ) and raccoons (8 of 11) participated in the study and received an average of nine testing sessions (range $=6-12$ ). Despite strong efforts to habituate coyotes to the experimental setup and motivate their participation, we only had one of six coyotes (male, Orion) participate in trials. With the exception of one coyote that was removed from testing prematurely due to stress (female, Vela), all other coyote subjects received an average of 55 testing days (range $=39-88$ ) and 40 testing sessions (range $=26-65$ ), during which they approached and touched the device at least once. Ultimately, we had a total sample size of 12 subjects across species (skunks: $n=3$, raccoons: $n=8$, coyotes: $n=1$ ) the majority of which had at least some prior experience (skunks: A: $n=1, \mathrm{~B}: n=1$, C: $n=1$; raccoons: A: $n=3, \mathrm{~B}: n=2, \mathrm{C} n=3$; coyotes: A: $n=1, \mathrm{~B}: n=0, \mathrm{C}: n=0)$. Subjects varied in the number of pre-reversals (range $=6-12$ ) and reversals (range $=0-32$ ) completed (Table 1). We experienced two instances of unexpected device malfunction during raccoon testing (once with Pollux and once with Rigel) and did not include data from these two instances in our analyses (see ESM).

Raccoons typically began interacting with the device during their first or second testing session (Fig. 2), completed 
Table 1 Individual traits and variation in performance of study subjects

\begin{tabular}{|c|c|c|c|c|c|c|c|c|c|}
\hline Name & Species & Sex & Origin & $\begin{array}{l}\text { Prior expe- } \\
\text { rience }\end{array}$ & $\begin{array}{l}\text { Number of pre- } \\
\text { reversals }\end{array}$ & $\begin{array}{l}\text { Number of } \\
\text { reversals }\end{array}$ & $\begin{array}{l}\text { Side bias } \\
\text { reversals }\end{array}$ & WSLS blocks & $\begin{array}{l}\text { Reversal } \\
\text { improve- } \\
\text { ment* }\end{array}$ \\
\hline Neptune & Skunk & M & $\mathrm{CB}$ & $\mathrm{A}$ & 12 & 13 & No & 0 & No \\
\hline Saturn & Skunk & M & $\mathrm{CB}$ & $\mathrm{B}$ & 10 & 12 & No & 2 & Yes \\
\hline Jupiter & Skunk & M & $\mathrm{CB}$ & $\mathrm{C}$ & 6 & 2 & NA & 0 & NA \\
\hline Mars & Skunk & M & $\mathrm{CB}$ & $\mathrm{C}$ & 0 & 0 & NA & NA & NA \\
\hline Luna & Raccoon & $\mathrm{F}$ & WC & A & 8 & 4 & NA & NA & NA \\
\hline Rigel & Raccoon & M & WC & A & 6 & 20 & Left & 5 & Yes \\
\hline Sirius & Raccoon & M & WC & $\mathrm{A}$ & 9 & 23 & No & 0 & Yes \\
\hline Pollux & Raccoon & M & WC & $\mathrm{B}$ & 12 & 32 & No & 4 & Yes \\
\hline Vega & Raccoon & $\mathrm{F}$ & WC & B & 9 & 32 & No & 5 & Yes \\
\hline Astrid & Raccoon & $\mathrm{F}$ & WC & $\mathrm{C}$ & 12 & 13 & No & 0 & Yes \\
\hline Castor & Raccoon & M & WC & $\mathrm{C}$ & 9 & 31 & Left & 0 & Yes \\
\hline Celeste & Raccoon & $\mathrm{F}$ & WC & $\mathrm{C}$ & 0 & 0 & NA & NA & NA \\
\hline Nova & Raccoon & $\mathrm{F}$ & WC & $\mathrm{C}$ & 0 & 0 & NA & NA & NA \\
\hline Oberon & Raccoon & M & WC & $\mathrm{C}$ & 11 & 28 & No & 0 & Yes \\
\hline Titan & Raccoon & M & WC & $\mathrm{C}$ & 0 & 0 & NA & NA & NA \\
\hline Orion & Coyote & M & WC & A & 12 & 9 & No & 2 & No \\
\hline Cepheus & Coyote & M & $\mathrm{CB}$ & B & 0 & 0 & NA & NA & NA \\
\hline Draco & Coyote & M & WC & $\mathrm{C}$ & 0 & 0 & NA & NA & NA \\
\hline Leo & Coyote & M & $\mathrm{CB}$ & $\mathrm{C}$ & 0 & 0 & NA & NA & NA \\
\hline Perseus & Coyote & M & $\mathrm{CB}$ & $\mathrm{C}$ & 0 & 0 & NA & NA & NA \\
\hline Vela & Coyote & $\mathrm{F}$ & $\mathrm{CB}$ & $\mathrm{C}$ & 0 & 0 & NA & NA & NA \\
\hline
\end{tabular}

Sex: $\mathrm{M}=$ male, $\mathrm{F}=$ female; Origin: $\mathrm{WC}=$ wild-caught, $\mathrm{CB}=$ captive-born; $\mathrm{NA}=$ column not applicable to the individual

Prior Experience: $\mathrm{A}=$ moderate prior experience, $\mathrm{B}=$ low prior experience, $\mathrm{C}=$ no prior experience

WSLS blocks $=$ Number of win stay-lose shift blocks (i.e., only 1 error made per block) performed in reversals

*Improvement determined by $P$ values and confidence intervals (see SI)

Fig. 2 Maximum number of testing sessions allocated to skunks $(n=4)$, raccoons $(n=11)$, and coyotes $(n=6)$. Black lines indicate the number of testing sessions administered before participants began interacting with the testing device (averages presented for raccoons and skunks). Although raccoon and skunk participants began interacting within the first few nights, Orion did not begin engaging with the device until his 44th testing session

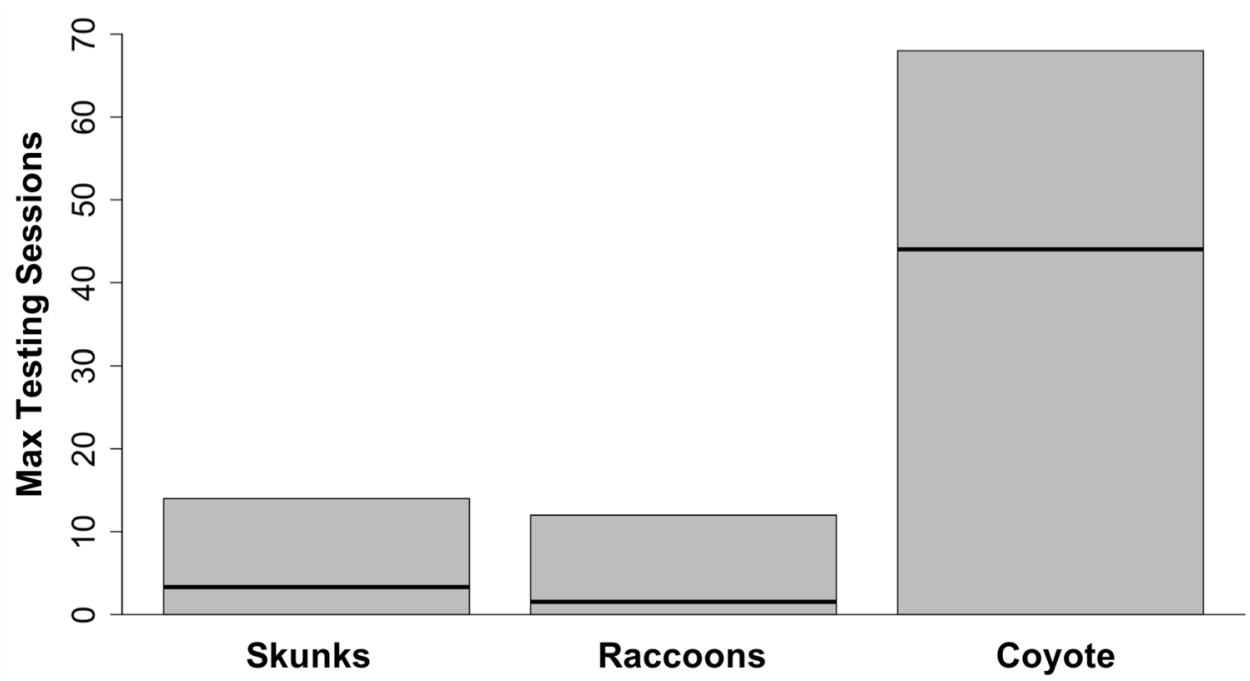

an average of 2.4 reversals during each testing session (range $=0-6$ ), and completed an average of 27 reversals in total (range $=4-32$ ). Skunks also began interacting with the device within their first few testing sessions (Fig. 2) but completed an average of 0.8 reversals per testing session (range $=0-2$ ) and an average of nine reversals in total 
(range $=0-13$ ). Our single coyote participant did not begin actively pushing on the pedals of the device until the 44th night (Fig. 2) and, similar to the skunks, typically achieved 0.5 reversals per testing session (range $=0-2$ ) and completed nine reversals in total (Table 1).

Our analysis was limited by the number of individuals per species that participated in the study. Nevertheless, because we observed some behavioral differences among species in response to testing, and because our protocol varied slightly between the two testing facilities, we proceeded with investigation of the number of errors made by species groups (in addition to other factors that could explain variation in performance), but did so with caution. Our top model investigating predictors of performance included both reversal number and individual ID as fixed effects (see ESM Table 2 for all model selection results) and indicated that subjects improved across reversals $(\beta=-0.05,95 \%$ $\mathrm{CI}=-0.06,-0.04, P<0.001$ ) (Fig. 3). Reversal number alone explained $26 \%$ of the variation in number of errors. Individual ID and prior experience explained an additional $16 \%$ and $11 \%$ (respectively) of the variation in the number of errors when included as fixed effects in the model alongside reversal number. In contrast, species identity explained only $2 \%$ of the variation in number of errors when included as a fixed effect in the model alongside reversal number, although again, this result should be interpreted with caution given our low sample size within each species group. In visually comparing the $y$-intercepts of each subject's learning curve (Fig. 3a), animals with no prior experience made a higher number of errors initially in reversals (Fig. 4b). However, no obvious patterns regarding prior experience emerged when visually comparing each subject's slope (Fig. 3c). We also did not observe any obvious patters with regards to species in visual review of learning curves (Fig. 3a-c).

Poisson regression performed individually for each subject suggested that the majority of subjects improved across trials and thus was not constrained to groups with or without prior experience (Table 1; all individual models and accompanying metrics in ESM Table 3). One skunk (Saturn) and seven raccoons (Astrid, Castor, Oberon, Pollux, Rigel, Sirius, and Vega) showed improvement in reversals. In contrast, our single coyote participant (Orion) and one skunk (Neptune) did not show improvement. Except for one raccoon (Pollux), none of the subjects exhibited a decrease in the number of blocks needed to meet the learning criterion. Instead, we found that subjects typically required a low number of blocks to meet criterion in reversals (skunks: mean $=2.4$, range $=1-4$; raccoons: mean $=2.3$, range $=1-6$;

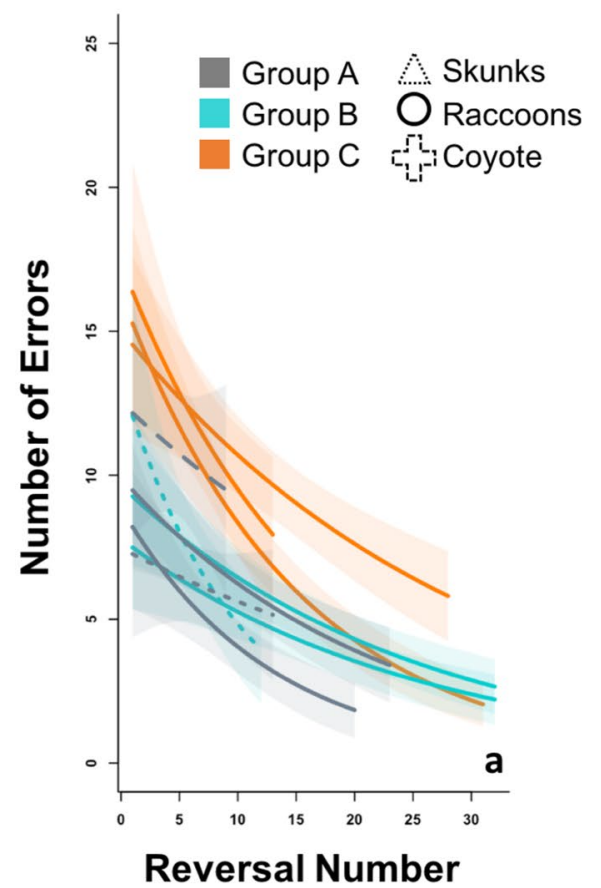

Fig. 3 Learning curves ( \pm SE) for each study participant during the reversal phase based on generalized linear models of individual performance (a). Panel (b) ranks the $y$-intercept of each participant's learning curve from the highest number of initial errors to the lowest number of initial errors made. Panel $\mathbf{c}$ ranks the slope of each learning curve from the steepest, indicating the greatest improvement made, to the flattest slope, indicating the least amount of improve-

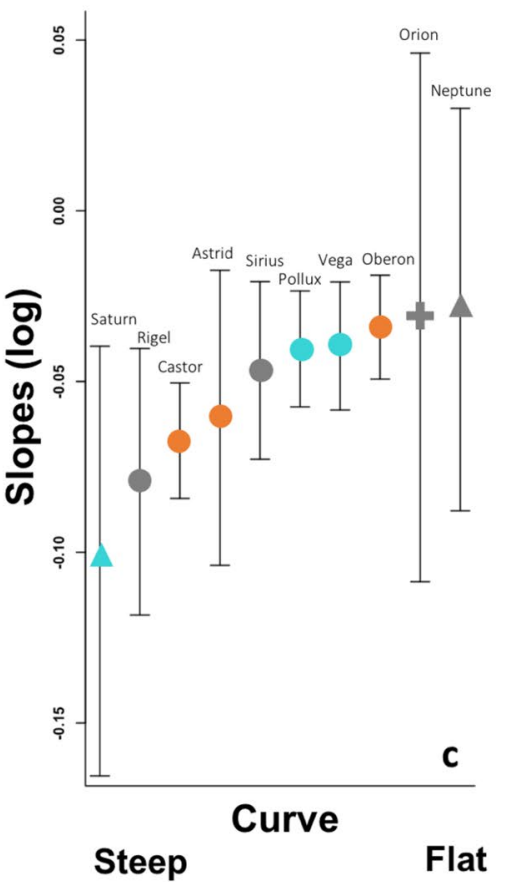

ment. Reversal curves suggest that animals with no prior experience made a higher number of initial errors, but no clear pattern emerges with regards to species. Subjects are identified by species (line type and symbol $)$ and level of prior experience $(\mathrm{A}=$ moderate prior experience, gray; $\mathrm{B}=$ low prior experience, blue; $\mathrm{C}=$ no prior experience, orange) (color figure online) 

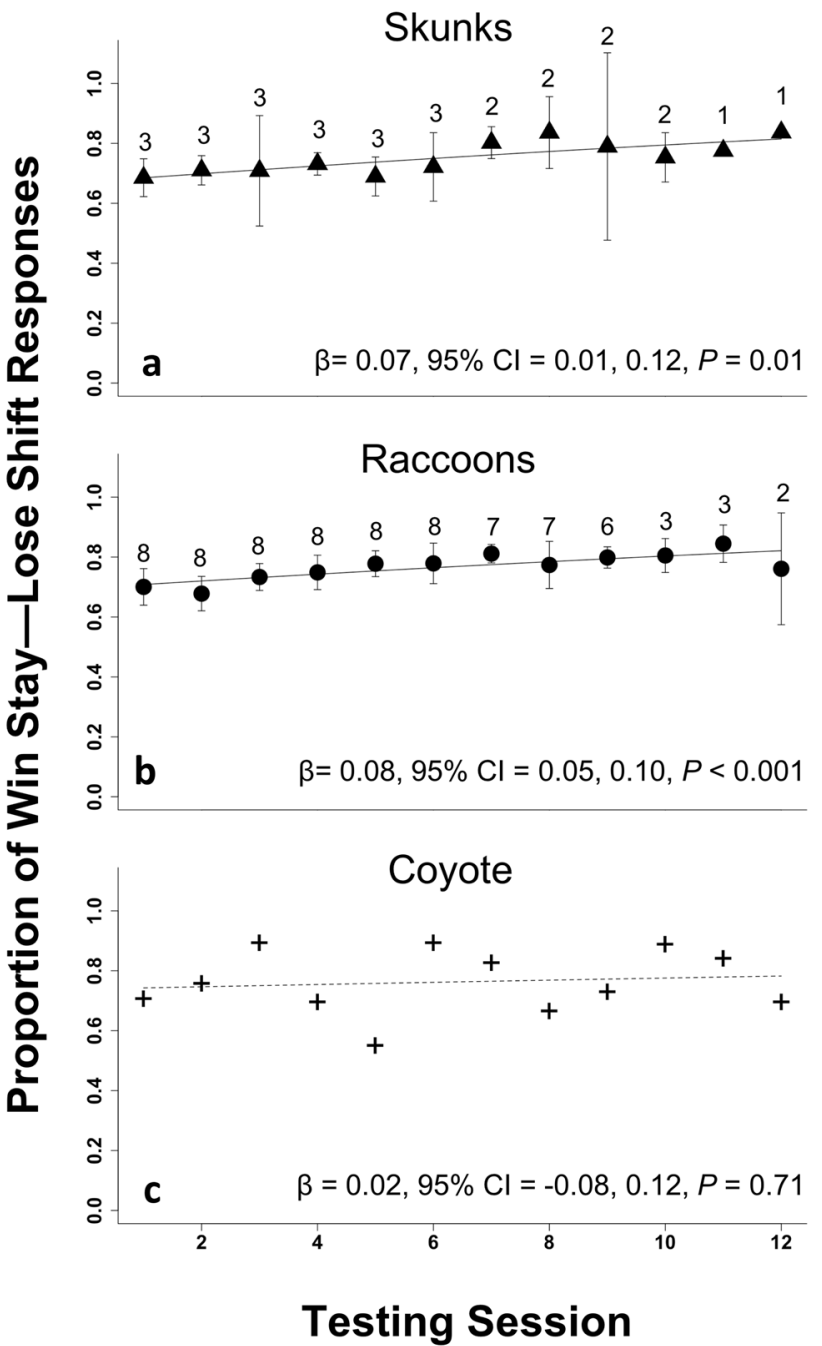

Fig. 4 Win stay-lose shift analysis. Average proportion $( \pm S E)$ of choice strategies employed by a skunk, $\mathbf{b}$ raccoon, and $\mathbf{c}$ coyote subjects across testing sessions with predicted linear model (includes data from all pre-reversals and reversals). Subjects primarily demonstrated a win stay strategy (60\% of all choices) and win stay-lose shift increased slightly across trials for skunks and raccoons. The sample size (number of participants) per testing session is indicated (note: only one coyote participant)

coyote mean $=3.3$, range $=1-6$ ). The results of our side bias analyses suggested that two of our raccoon subjects (Rigel and Castor) may have had a left-side bias, but this did not inhibit their ability to improve in reversals (Table 1). One skunk (Jupiter) and one raccoon (Luna), only completed two and four reversals, respectively, and consequently had an insufficient amount of data to evaluate individual learning curves (see ESM).

With regards to the sequence of choices that subjects made across testing sessions, we found that win stay was the most common strategy ( $60 \%$ of all choices made by subjects). The proportion of win stay-lose shift responses increased across trials for raccoons $(\beta=0.08,95 \% \mathrm{CI}=0.05$,
$0.10, P<0.001)$ and skunks $(\beta=0.07,95 \% \mathrm{CI}=0.01,0.12$, $P=0.01$ ), whereas Orion's performance varied across testing sessions and did not demonstrate an increase in win stay-lose shift responses (quasibinomial GLM with logit link: $\beta=0.02,95 \% \mathrm{CI}=-0.08,0.12, P=0.71$ ) (Fig. 4). However, Orion, along with Saturn (skunk), Pollux, Riegel, and Vega (raccoons), demonstrated a win stay-lose shift strategy (i.e., only one error made) multiple times in reversal testing (Table 1), albeit infrequent and not always repeated in succession.

\section{Discussion}

Behavioral plasticity is expected to play an important role in the ability of animals to adapt to environmental variation (Mettke-Hofmann 2014) and is, therefore, likely to bolster their success in urban habitats (Ducatez et al. 2018). Here we measured the cognitive flexibility of three generalist mesocarnivores that are commonly found in urban habitats. All subjects across species typically required a low number of blocks (i.e., 2-3) to meet the $90 \%$ learning criterion and most demonstrated an ability to form and reverse associations. However, we observed differences in the response of subjects to testing and found moderate individual variation in reversal learning performance. This variation may be partially explained by an individual's prior experience with the task, yet it did not affect an individual's ability to improve their reversal learning across time. Intraspecific differences in cognitive flexibility may be also affected by traits outside of the scope of our study, including sex (Lucon-Xiccato and Bisazza 2014; Petrazzini et al. 2017), age (Johnson and Wilbrecht 2011; Tello-Ramos et al. 2018), temperament (e.g., bold vs. shy individuals; Mazza et al. 2018), and origin (Croston et al. 2017). Therefore, future research on the reversal-learning abilities of mesocarnivores would benefit from investigating the drivers of individual variation in testing performance among a single species with an increased sample size.

Raccoons generally showed engagement and success in this task. Most raccoons demonstrated rapid associative learning in that they learned to use the testing device and began passing blocks within their first testing session. Similarly, most raccoons completed several reversals and showed trends for improvement in reversal learning across time. These results join a growing body of literature that demonstrates the cognitive flexibility of raccoons and supports the notion that cognition and behavioral plasticity enables the heightened efficiency of raccoons to exploit urban habitats. For example, wild raccoons are able to solve novel foraging challenges associated with urban living (e.g., open garbage bins; MacDonald and Rivto 2016), demonstrate plasticity in social behavior (Prange 
and Gehrt 2004; Prange et al. 2011), and have heightened opportunities for transmission of information in comparison to other urban mesocarnivores (e.g., group foraging, extended familial bonds, Gehrt 2004). Recent research employing the Aesop's Fable paradigm (Stanton et al. 2017) and multi-access puzzle boxes (Daniels et al. 2019) further confirms that raccoons can learn rapidly and are flexible in their problem-solving behavior.

Although our sample size for skunks was limited, we found that our skunk participants were willing to approach and engage with the device rather quickly. Nevertheless, they did not complete a high number of reversals and only one of three skunks demonstrated a trend towards improvement. Given their small body size and amount of reward received upon correct selections, we suspect that this deficit in performance may reflect a lack of food motivation and/or rapid satiation by skunks, rather than a particular learning barrier. For instance, skunks made a low number of errors in reversals and demonstrated a trend towards a win staylose shift strategy. In addition, our results generally align with the findings of Gossette et al. (1968) who reported that the reversal-learning performance of skunks, raccoons, and other mesocarnivores was similar. Because the cognition of skunks is generally under-investigated (but see Vonk and Leete 2017; Johnson-Ulrich et al. 2017), further research on the cognition of skunks, and how it relates to their urban adaptation, is currently needed. Additional comparative investigations of raccoon and skunk cognition may prove especially interesting because of the differences in their brain morphology (e.g., low relative brain size in skunks, high relative brain size and neuronal density in raccoons; Jardim-Messeder et al. 2017; Stankowich and Romero 2017), but this will require larger sample sizes of each species, as well as additional evidence linking mesocarnivore brain morphology with cognitive capacity (e.g., brain size and problem-solving ability; Benson-Amram et al. 2016).

Our results for coyotes are more ambiguous due to a low participation rate for this species. Although our single coyote participant, Orion, was capable of completing reversals, demonstrated a win stay-lose shift strategy in two of his reversals, and generally made a low number of errors, he exhibited wavering participation and a lack of improvement in testing. Captive coyotes at the same testing facility demonstrated behavioral plasticity in a similar operant conditioning study (Gilbert-Norton et al. 2009) and completed a problem-solving task with puzzle boxes (Young et al. 2019b). Moreover, wild, urban coyotes have shown behavioral plasticity in diet composition (Newsome et al. 2015) and activity patterns (Murray and St. Clair 2015). We, therefore, suspect that Orion's lack of improvement and the low participation by coyotes in this study may have been related to motivation and object neophobia, rather than a particular learning barrier or a lack of inhibitory control.
Neophobia towards the testing device in our study was not unexpected, because coyotes are generally known to be cautious of novel objects (Mettler and Shivik 2007; Windberg, 2008). However, we were surprised to find that captive coyotes continued to demonstrate neophobia toward our automated testing device despite our extensive efforts to habituate them to the experimental procedure. Although captive coyotes have been successfully habituated to testing apparatuses when a human investigator was involved and have demonstrated learning and flexibility in empirical studies of cognition (Gilbert-Norton et al. 2009; Young et al. 2019b), captive coyotes have also shown a lack of habituation to frightening devices that use a combination of light and sound stimuli (Darrow and Shivik 2009). Indeed, managers and livestock owners are known to employ these stimuli in conflict mitigation scenarios to successfully deter predation on livestock (e.g., Zarco-González and MonroyVilchis 2014; Lesilau et al. 2018). Similarly, in a recent problem-solving study, a puzzle box required modification to reduce the startle response by captive coyotes related to the noise made during door removal (Young et al. 2019b). The LED lights and motor-dispensing sound of our testing device also appeared to have startled the coyotes and may, therefore, explain why most of the coyotes never interacted with the device, despite showing interest and attempting to remove food from it (see ESM video 4 for example). Therefore, we recommend that future studies employing automated cognitive testing devices with coyotes reduce any unnecessary light and sound stimuli.

Although generalists may gain benefits from reduced neophobia and greater exploration, neophobia may also serve to protect individuals from the unknown potential dangers of new things (sensu the "Dangerous Niche Hypothesis", Greenberg 2003). In this way, coyotes may resemble other successful cosmopolitan generalists that show heightened aversion to novelty, such as rats (Rattus spp.) and corvids (Corvus spp.) because of their historic persecution by humans (Greenberg and MettkeHofmann 2001; Vernelli 2013; Greggor et al. 2016). However, unlike rats and corvids, urbanization may be relaxing pressure on coyotes and could explain why current research is revealing increased boldness in urban vs. rural coyotes (Schell et al. 2018, 2020; Breck et al. 2019; Brooks et al. 2020). Similarly, mixed findings on the cognitive and behavioral competencies of urban wildlife might imply that different cities impose different inter and intraspecific pressures, and that this may be sensitive not only to the natural history of a species, but also the degree of conflict animals face with the local human population (Griffin et al. 2017; Kozlovsky et al. 2017; Barrett et al. 2019; Schell et al. 2020). Indeed, enhanced cognition and behavior plasticity may be one of many strategies that facilitate exploitation of urban environments (Santini 
et al. 2019; Sayol et al. 2020). Therefore, although cognition likely acts as a buffer (Sol 2009), we might expect diversification of the cognitive and behavioral strategies generalists use to exploit urban environments; some of which may demand more cognitive complexity, whereas others may be more cognitively simple (Davey 1989; McKinney 2006; Kozlovsky et al. 2017). In our study, we found only moderate individual variation in the number of errors made (i.e., explained $16 \%$ of variation), which may indicate similar levels of cognitive flexibility; however, we also observed behavioral differences among individuals and species that warrant further investigation. Experimental investigation of cognition in wild populations will continue to be invaluable in understanding urban adaptation and the co-evolutionary forces between humans and urban wildlife (Barrett et al. 2019; Schell et al. 2020).

Our study was limited by a small sample size of participants. Because we were unable to systematically encourage participation (e.g., food deprivation), we relied heavily on the interest and self-motivation of our subjects. Given this limitation, we were unable to generate enough subjects across traits of interest (e.g., sex, age, temperament, origin) to identify how these traits may have affected reversal-learning performance, and in most cases our analyses were limited in power. Furthermore, our protocol evolved over time as we gained experience working with these animals that have yet to be tested in this manner and, therefore, includes individuals that varied in prior testing experience. Thus, some of our results should be interpreted with caution. Nevertheless, the species-specific accommodations made during testing allowed us to adapt our methodology as necessary. Our study has, therefore, made important contributions to the field of comparative cognition and has expanded our ability to investigate the cognitive underpinnings of urban adaptation in a greater diversity of species. Automated protocols and testing devices, such as the one developed in our study and similar studies in birds (e.g., Bridge et al. 2019), remove the role of an experimenter and better enables research on cognition in the wild (Morand-Ferron, Cole, et al. 2015a, b), which is an important next step in understanding the adaptability of urban carnivores. Because carnivores represent an understudied yet diverse group of generalists and specialists that differ in their use of anthropogenic areas, including procyonids (e.g., raccoons vs. crab-eating raccoons (Procyon cancrivorus)), felids (e.g., bobcats (Lynx rufus) vs. Canada lynx (Lynx canadensis)), and ursids (e.g., black bears (Ursus americanus) vs. sun bears (Helarctos malayanus) or giant pandas (Ailuropoda melanoleuca)), this group can offer new insights in animal cognition and urban adaptation.

\section{Conclusion}

Our study offers some support for the hypothesis that urban mesocarnivores are cognitively, and thereby behaviorally, flexible based on their performance in a classic reversal learning paradigm. Behavioral plasticity is expected to underlie the ability of generalists to persist in challenging environments, including urban habitats, where animals may be encountering novel, complex, and changing stimuli. The demonstrated ability of our subjects to form and reverse learned associations based on the cues of the paradigm suggests that they have the capacity to change their behavior in a flexible manner; however, there may be variation in this flexibility. Testing of wild individuals across urban-rural gradients, as well as in urban populations representing different human attitudes and behaviors towards wildlife, will be essential for linking flexibility in cognition to urban adaptation. Furthermore, variation in willingness of subjects to engage with novelty in this experiment serves as a reminder of the inter and intraspecific differences in exploration, and how such differences may reflect the different strategies of behavioral adaptation to urban habitats, even within a single group like generalists. Our study is an important first step in advancing current methodologies for the study of less-traditional species, and we expect that future studies of the cognition of wild mesocarnivores will benefit from automated testing devices like the one deployed in our study. Such contributions to our understanding of adaptation to urban habitats will advance our ability to mitigate humanwildlife conflict, conserve biodiversity in urban habitats, and elucidate the evolutionary trajectory of cognition in the Anthropocene.

Acknowledgements We would like to thank many people who contributed their expertise to the development of this study, including: Joel Fagot, Lynne Gilbert-Norton, Lydia Hopper, Sarah Jacobson, Zoe Johnson-Ulrich, Glenn Tattersall, Raymond Vagell, Josh Van Bourg, and Jason Watters. We also thank Steve DeVries for the construction of our testing devices, as well as Brett Jesmer and the Animal Behavior and Cognition Lab for their assistance with statistical analyses and manuscript feedback. Lastly, we thank NWRC personnel for their assistance conducting this study, specifically: Stacey Brummer, Mike Davis, Nate Floyd, Amy Gilbert, Buck Jolley, Dimitri Latimir, Austin Merical, Jeff Schultz, and Chad Wickham. The findings and conclusions in this publication have not been formally disseminated by the U.S. Department of Agriculture and should not be construed to represent any agency determination or policy. This work was supported by the intramural research program of the U.S. Department of Agriculture, National Wildlife Research Center, as well as the Wyoming NASA Space Grant Consortium Fellowship (NASA Grant No. NNX10AO95H) and Berry Ecology Research Grant awarded to L.A.S.

Funding This work was supported by the intramural research program of the U.S. Department of Agriculture, National Wildlife Research Center, as well as the Wyoming NASA Space Grant Consortium Fellowship (NASA Grant No. NNX10AO95H) and the University of Wyoming Berry Ecology Research Grant awarded to L.A.S. 
Data availability Data is available at https://bit.ly/31XbTFK.

Code availability Code is available at https://bit.ly/31XbTFK.

\section{Compliance with ethical standards}

Conflict of interest The authors declare that they do not have any conflict of interest.

Ethics approval This study was approved by the USDA National Wildlife Research Center's Institute for Animal Care and Use Committee (QA-2825).

\section{References}

Alberti M, Correa C, Marzluff JM, Hendry AP, Palkovacs EP, Gotanda KM (2016) Global urban signatures of phenotypic change in animal and plant populations. Proc Natl Acad Sci USA 114(34):1-6

Anderson MC, Neeley JH (1996) Interference and inhibition in memory retrieval. Memory (Handbook of perception and cognition). Academic Press, San Diego, pp 237-313

Ashton BJ, Ridley AR, Edwards EK, Thornton A (2018) Cognitive performance is linked to group size and affects fitness in Australian magpies. Nature 554(7692):364-367

Audet J-N, Lefebvre L (2017) What's flexible in behavioral flexibility? Behav Ecol 28(4):943-947

Baker JM, Shivik J, Jordan KE (2011) Tracking of food quantity by coyotes (Canis latrans). Behav Processes 88(2):72-75

Barrett LP, Stanton L, Benson-Amram S (2019) The cognition of 'nuisance' species. Anim Behav 147:167-177

Bartoń K (2018) MuMIn: Multi-Model Inference. R package version 1.42.1. https://CRAN.R-project.org/package=MuMIn

Bateman PW, Fleming PA (2012) Big city life: carnivores in urban environments. J Zool 287(1):1-23

Bates D, Mächler M, Bolker B, Walker S (2015) Fitting linear mixedeffects models using lme4. J Stat Softw 67(1):1-48. https://doi. org/10.18637/jss.v067.i01

Benson-Amram S, Dantzer B, Stricker G, Swanson EM, Holekamp KE (2016) Brain size predicts problem-solving ability in mammalian carnivores. Proc Natl Acad Sci 113(9):2532-2537

Bond AB, Kamil AC, Balda RP (2007) Serial reversal learning and the evolution of behavioral flexibility in three species of North American corvids (Gymnorhinus cyanocephalus, Nucifraga columbiana, Aphelocoma californica). J Comp Psychol 121(4):372-379

Breck SW, Poessel SA, Mahoney P, Young JK (2019) The intrepid urban coyote: a comparison of bold and exploratory behavior in coyotes from urban and rural environments. Sci Rep 9:1-11

Bridge ES, Wilhelm J, Pandit MM, Moreno A, Curry CM, Pearson TD, Proppe DS, Holwerda C, Eadie JM, Stair TF et al (2019) An Arduino-based RFID platform for animal research. Front Ecol Evol 7(July):1-10

Bridgeman JM, Tattersall GJ (2019) Tortoises develop and overcome position biases in a reversal learning task. Anim Cogn 22(2):265-275

Brooks ME, Kristensen K, van Benthem KJ, Magnusson A, Berg CW, Nielsen A, Skaug HJ, Maechler M, Bolker BM (2017) glmmTMB balances speed and flexibility among packages for zero-inflated generalized linear mixed modeling. The R J 9(2):378-400
Brooks J, Kays R, Hare B (2020) Coyotes living near cities are bolder: implications for dog evolution and human-wildlife conflict. Behaviour 1(aop):1-25

Buechel SD, Boussard A, Kotrschal A, van Der Bijl W, Kolm N (2018) Brain size affects performance in a reversal-learning test. Proc $\mathrm{R}$ Soc B Biol Sci. https://doi.org/10.1098/rspb.2017.2031

Cauchoix M, Hermer E, Chaine AS, Morand-Ferron J (2017) Cognition in the field: comparison of reversal learning performance in captive and wild passerines. Sci Rep 7(1):12945

Chow PKY, Leaver LA, Wang M, Lea SEG (2015) Serial reversal learning in gray squirrels: learning efficiency as a function of learning and change of tactics. J Exp Psychol Anim Learn Cogn 41(4):343-353

Croston R, Branch CL, Pitera AM, Kozlovsky DY, Bridge ES, Parchman TL, Pravosudov VV (2017) Predictably harsh environment is associated with reduced cognitive flexibility in wild food-caching mountain chickadees. Anim Behav 123:139-149

Daniels SE, Fanelli RE, Gilbert A, Benson-Amram S (2019) Behavioral flexibility of a generalist carnivore. Anim Cogn 22(3):387-396

Darrow PA, Shivik JA (2009) Bold, shy, and persistent: Variable coyote response to light and sound stimuli. Appl Anim Behav Sci 116(1):82-87

Davey G (1989) Ecological learning theory. Routledge, London

Devictor V, Julliard R, Jiguet F (2008) Distribution of specialist and generalist species along spatial gradients of habitat disturbance and fragmentation. Oikos 117(4):507-514

Doty BA, Combs WC (1969) Reversal learning of object and positional discriminations by mink, ferrets and skunks. Q J Exp Psychol 21(1):58-62

Ducatez S, Clavel J, Lefebvre L (2015) Ecological generalism and behavioural innovation in birds: technical intelligence or the simple incorporation of new foods? J Anim Ecol 84(1):79-89

Ducatez S, Sayol F, Sol D, Lefebvre L (2018) Are urban vertebrates city specialists, artificial habitat exploiters, or environmental generalists? Integr Comp Biol 58(5):929-938

Fields PE (1936) A comparison of white rats and raccoons with respect to their visual discrimination of certain geometrical figures. J Comp Psychol 21:341-355

Gehrt SD (2004) Ecology and management of striped skunks, raccoons, and coyotes in urban landscapes. In: Fascione N, Delach A, Smith M (eds) People and predators: from conflict to coexistence. Island Press, Washington, pp 81-104

Gehrt SD, Riley SPD, Cypher BL (2010) Urban carnivores: ecology, conflict, and conservation. JHU Press, Baltimore

Gilbert-Norton LB, Shahan TA, Shivik JA (2009) Coyotes (Canis latrans) and the matching law. Behav Processes 82(2):178-183

Godfrey-Smith P (1996) Complexity and the function of mind in nature. Cambridge University Press, Cambridge

Gossette RL, Kraus G, Speiss J (1968) Comparison of successive discrimination reversal (SDR) performances of seven mammalian species on a spatial task. Psychon Sci 12(5):193-194

Greenberg R (2003) The role of neophobia and neophilia in the development of innovative behaviour of birds. In: Reader SM, Laland KN (eds) Animal innovation. Oxford University Press, Oxford, pp 175-196

Greenberg R, Mettke-Hofmann C (2001) Current ornithology, Volume 16. In: Nolan VJ, Thompson CF (eds) Current ornithology, vol 16. Kluwer Academic/Plenum Publishers, New York, pp 119-178

Greggor AL, Clayton NS, Fulford AJC, Thornton A (2016) Street smart: faster approach towards litter in urban areas by highly neophobic corvids and less fearful birds. Anim Behav 117:123-133

Griffin AS, Netto K, Peneaux C (2017) Neophilia, innovation and learning in an urbanized world: a critical evaluation of mixed findings. Curr Opin Behav Sci 16:15-22. https://doi.org/10.1016/J. COBEHA.2017.01.004 
Grimm NB, Faeth SH, Golubiewski NE, Redman CL, Wu J, Bai X, Briggs JM (2008) Global change and the ecology of cities. Science (80-) 319(5864):756-760

Hermer E, Cauchoix M, Chaine AS, Morand-Ferron J (2018) Elevation-related difference in serial reversal learning ability in a nonscatter hoarding passerine. Behav Ecol 29(4):840-847

Holekamp KE, Benson-Amram S (2017) The evolution of intelligence in mammalian carnivores. Interface Focus. 7(3):20160108

Izquierdo A, Brigman JL, Radke AK, Rudebeck PH, Holmes AA (2017) Review the neural basis of reversal learning: an updated perspective. Neuroscience 345:12-26

Jardim-Messeder D, Lambert K, Noctor S, Pestana FM, de Castro Leal ME, Bertelsen MF, Alagaili AN, Mohammad OB, Manger PR, Herculano-Houzel S (2017) Dogs have the most neurons, though not the largest brain: trade-off between body mass and number of neurons in the cerebral cortex of large carnivoran species. Front Neuroanat 11:118

Johnson C, Wilbrecht L (2011) Juvenile mice show greater flexibility in multiple choice reversal learning than adults. Dev Cogn Neurosci 1(4):540-551

Johnson JI, Michels KM (1958) Learning sets and object-size effects in visual discrimination learning by raccoons. J Comp Physiol Psychol 51(3):376-379

Johnson MTJ, Munshi-South J (2017) Evolution of life in urban environments. Science 358(6363): eaam8327

Johnson-Ulrich Z, Hoffmaster E, Robeson A, Vonk J (2017) Visual acuity in the striped skunk (Mephitis mephitis). J Comp Psychol 131(4):384

Kozlovsky DY, Weissgerber EA, Pravosudov VV (2017) What makes specialized food-caching mountain chickadees successful city slickers? Proc R Soc B Biol Sci 284(1855):20162613

Lai ZC, Moss MB, Killiany RJ, Rosene DL, Herndon JG, Lai ZC, Moss MB, Killiany RJ, Rosene DL, Herndon JG (1995) Executive system dysfunction in the aged monkey: spatial and object reversal learning. Neurobiol Aging. 16(95):947-956

Lefebvre L, Whittle P, Lascaris E, Finkelstein A (1997) feeding innovations and forebrain size in birds. Anim Behav 53:549-560

Lesilau F, Fonck M, Gatta M et al (2018) Effectiveness of a LED flashlight technique in reducing livestock depredation by lions (Panthera leo) around Nairobi National Park, Kenya. PLoS ONE 13:1-18

Liu Y, Day LB, Summers K, Burmeister SS (2016) Learning to learn: advanced behavioural flexibility in a poison frog. Anim Behav 111:167-172

Lowry H, Lill A, Wong BBM (2013) Behavioural responses of wildlife to urban environments. Biol Rev 88(3):537-549

Lucon-Xiccato T, Bisazza A (2014) Discrimination reversal learning reveals greater female behavioural flexibility in guppies. Biol Lett 10(6):20140206

MacDonald SE, Ritvo S (2016) Comparative cognition outside the laboratory. Comp Cogn Behav Rev. https://doi.org/10.3819/ ccbr.2016.110003

Mackintosh NJ, McGonigle B, Holgate V, Vanderver V (1968) Factors underlying improvement in serial reversal learning. Can $\mathrm{J}$ Psychol 22(2):85-95

Macphail EM, Bolhuis JJ (2001) The evolution of intelligence: adaptive specializations versus general process. Biol Rev Camb Philos Soc 76(3):341-364

Madden JR, Langley EJG, Whiteside MA, Beardsworth CE, Van Horik JO (2018) The quick are the dead: pheasants that are slow to reverse a learned association survive for longer in the wild. Philos Trans R Soc B Biol Sci. https://doi.org/10.1098/ rstb.2017.0297

Mahamane S, Grunig KL, Baker J, Young JK, Jordan KE (2014) Memory-based quantity discrimination in coyotes (Canis latrans). Anim Behav Cogn 1(3):341
Maklakov AA, Immler S, Gonzalez-voyer A, Rönn J, Kolm N, Supplement D (2011) Brains and the city: big-brained passerine birds succeed in urban environments. Biol Lett 7(April):730-732

Mazza V, Eccard JA, Zaccaroni M, Jacob J, Dammhahn M (2018) The fast and the flexible: cognitive style drives individual variation in cognition in a small mammal. Anim Beh 137(March):119-132

McKinney ML (2002) Urbanization, biodiversity, and conservation. Bioscience 52(10):883-890

McKinney ML (2006) Urbanization as a major cause of biotic homogenization. Biol Conserv 127(3):247-260

Mettke-Hofmann C (2014) Cognitive ecology: ecological factors, life-styles, and cognition. Wiley Interdiscip Rev Cogn Sci. 5(3):345-360

Mettler AE, Shivik JA (2007) Dominance and neophobia in coyote (Canis latrans) breeding pairs. App Am Beh Sci 102(1-2):85-94

Morand-Ferron J (2017) Why learn? The adaptive value of associative learning in wild populations. Curr Opin Behav Sci 16:73-79

Morand-Ferron J, Cole EF, Quinn JL (2015a) Studying the evolutionary ecology of cognition in the wild: a review of practical and conceptual challenges. Biol Rev 91(2):367-389

Morand-Ferron J, Hamblin S, Cole EF, Aplin LM, Quinn JL (2015b) Taking the operant paradigm into the field: associative learning in wild great tits. PLoS ONE 10(8):1-16

Murray MH, St. Clair CC (2015) Individual flexibility in nocturnal activity reduces probability of road mortality for an urban carnivore. Beh Ecol 26:1520-1527

Navarrete A, Reader SM, Whalen A, Laland KN (2016) The co-evolution of innovation and technical intelligence in primates. Philos Trans R Soc B Biol Sci. https://doi.org/10.1098/rstb.2015.0186

Newsome SD, Garbe HM, Wilson EC, Gehrt SD (2015) Individual variation in anthropogenic resource use in an urban carnivore. Oecologia 178(1):115-128

Overington SE, Griffin AS, Sol D, Lefebvre L (2011) Are innovative species ecological generalists? A test in North American birds. Behav Ecol 22(6):1286-1293

Petrazzini MEM, Bisazza A, Agrillo C, Lucon-Xiccato T (2017) Sex differences in discrimination reversal learning in the guppy. Anim Cogn. 20:1081-1091

Pettit M (2010) The problem of raccoon intelligence in behaviourist America. Br J Hist Sci 43(03):391-421

Pimm SL, Jenkins CN, Abell R, Brooks TM, Gittleman JL, Joppa LN, Raven PH, Roberts CM, Sexton JO (2014) The biodiversity of species and their rates of extinction, distribution, and protection. Science 344(6187): 1246752

Prange S, Gehrt SD, Hauver S (2011) Frequency and duration of contacts between free-ranging raccoons: uncovering a hidden social system. J Mammal 92(6):1331-1342

Prange S, Gehrt SD (2004) Changes in mesopredator-community structure in response to urbanization. Can J Zool 82(11):1804-1817

R Core Team. 2018. R: A language and environment for statistical computing. https://www.r-project.org/

Robertson BA, Rehage JS, Sih A (2013) Ecological novelty and the emergence of evolutionary traps. Trends Ecol Evol 28(9):552-560

Santini L, González-Suárez M, Russo D, Gonzalez-Voyer A, von Hardenberg A, Ancillotto L (2019) One strategy does not fit all: determinants of urban adaptation in mammals. Ecol Lett 22(2):365-376

Sayol F, Sol D, Pigot AL (2020) Brain size and life history interact to predict urban tolerance in birds. Front Ecol Evol 8:1-9

Schell CJ, Young JK, Lonsdorf EV, Santymire RM, Mateo JM, Christopher Schell CJ (2018) Parental habituation to human disturbance over time reduces fear of humans in coyote offspring. Ecol Evol. https://doi.org/10.1002/ece3.4741

Schell CJ, Stanton LA, Young JK, Angeloni L, Lambert JE, Breck SW, Murray M (2020) The evolutionary consequences of 
human-wildlife conflict in cities. Evol Appl 00:1-20. https:// doi.org/10.1111/eva.13131

Seto KC, Güneralp B, Hutyra LR (2012) Global forecasts of urban expansion to 2030 and direct impacts on biodiversity and carbon pools. Proc Natl Acad Sci USA 109(40):16083-16088

Shell WF, Riopelle AJ (1957) Multiple discrimination learning in raccoons. J Comp Physiol Psychol 50(6):585-587

Shettleworth SJ (2010) Cognition, evolution, and behaviour, Second. Oxford University Press, New York

Sih A, Ferrari MCO, Harris DJ (2011) Evolution and behavioural responses to human-induced rapid environmental change. Evol Appl 4(2):367-387

Skinner BF (1938) The behavior of organisms: an experimental analysis. Appleton-Century, New York

Snell-Rood E, Wick N (2013) Anthropogenic environments exert variable selection on cranial capacity in mammals. Proc R Soc B Biol Sci 280(August): $1-10$

Sol D (2003) Behavioural innovation: a neglected issue in the ecological and evolutionary literature? In: Reader SM, Laland KN (eds) Animal innovation. Oxford University Press, Oxford, pp 63-82

Sol D (2009) Revisiting the cognitive buffer hypothesis for the evolution of large brains. Biol Lett 5(1):130-133

Sol D, Lefebvre L (2000) Behavioural flexibility predicts invasion success in birds introduced to New Zealand. Oikos 90(3):599-605

Sol D, Bacher S, Reader SM, Lefebvre L (2008) Brain size predicts the success of mammal species introduced into novel environments. Am Nat. 172(Suppl July 2008):S63-71

Sol D, Lapiedra O, Gonzalezlez-Lagos C (2013) Behavioural adjustments for a life in the city. Anim Behav 85(5):1101-1112

Sol D, Sayol F, Ducatez S, Lefebvre L (2016) The life history basis of behavioural innovations. Philos Trans R Soc B Biol Sci 371(1690):20150187

Sol D, Timmermans S, Lefebvre L (2002) Behavioural flexibility and invasion success in birds. Anim Behav 63(3):495-502

Stankowich T, Romero AN (2017) The correlated evolution of antipredator defences and brain size in mammals. Proc R Soc B Biol Sci 284(1846):20161857

Stanton L, Davis E, Johnson S, Gilbert A, Benson-Amram S (2017) Adaptation of the Aesop's Fable paradigm for use with raccoons
(Procyon lotor): considerations for future application in nonavian and non-primate species. Anim Cogn 20:1147-1152

Tebbich S, Teschke I (2014) Coping with uncertainty: Woodpecker Finches (Cactospiza pallida) from an unpredictable habitat are more flexible than birds from a stable habitat. PLoS ONE. https ://doi.org/10.1371/journal.pone.0091718

Tello-Ramos MC, Branch CL, Pitera AM, Kozlovsky DY, Bridge ES, Pravosudov VV (2018) Memory in wild mountain chickadees from different elevations: comparing first-year birds with older survivors. Anim Behav 137(February):149-160

Vernelli T (2013) The complexity of neophobia in a generalist foraging corvid: the common magpie (Pica pica), Thesis

Vonk J, Leete J (2017) Carnivore concepts: categorization in carnivores "bears" further study. Int J Comp Psychol 30:1-20

Warren JM, Warren HB (1962) Reversal learning by horse and raccoon. J Genet Psychol 100(2):215-220

Windberg LA (2008) Coyote responses to visual and olfactory stimuli related to familiarity with an area. Can J Zool 74(12):2248-2253

Wright TF, Eberhard JR, Hobson EA, Avery ML, Russello M (2010) Behavioral flexibility and species invasions: the adaptive flexibility hypothesis. Ethol Ecol Evol. 22(4):393-404

Young JK, Golla J, Draper JP, Broman D, Blankenship T, Heilbrun R (2019a) Space use and movement of urban bobcats. Animals 9(5):275

Young JK, Touzot L, Brummer SP (2019b) Persistence and conspecific observations improve problem-solving abilities of coyotes. PLoS ONE 14(7):e0218778

Zarco-González MM, Monroy-Vilchis O (2014) Effectiveness of lowcost deterrents in decreasing livestock predation by felids: a case in central Mexico. Anim Conserv 17:371-378

Publisher's Note Springer Nature remains neutral with regard to jurisdictional claims in published maps and institutional affiliations. 
Electronic Supplementary Material for "Variation in reversal learning by three generalist mesocarnivores"

\section{Animal subjects}

This study was approved by NWRC Institute for Animal Care and Use Committee (QA2825). Skunks (captive-bred from Ruby Fur Farm, New Sharon, IA) and raccoons (wild-caught from June and July 2017 in the Laporte, CO area) at the NWRC headquarters in Fort Collins, CO $\left(40^{\circ} 35^{\prime} \mathrm{N}, 105^{\circ} 05^{\prime} \mathrm{W}\right)$, were housed individually in outdoor pens $(3 \times 3 \times 2.5 \mathrm{~m})$. Each pen included a den box and various enrichment items, such as logs, paper bags, plastic balls, etc. Animals were fed Mazuri Omnivore-Zoo Feed 'A' (Richmond, IN); raccoons received 200g and skunks received $100 \mathrm{~g}$ daily, and water was available ad libitum. Coyotes at the 164-acre NWRC Predator Research Facility in Millville, UT are typically maintained as male-female pairs in outdoor enclosures (see Young et al. 2019 for more information). Most coyotes are born at the facility to captive parents, but wild-captured pups are obtained every few years to increase genetic diversity of the research colony, including our single successful coyote participant, Orion. These coyotes are hand-reared until 10-15 weeks of age and then maintained similarly to captive-born coyotes. Coyotes were fed six days a week with 650g of mink food (Fur Breeders Agricultural Cooperative Logan, UT) once a day with access to water ad libitum. All subjects were adults at the time of testing.

Skunks and raccoons were studied between the months of October 2017 and March 2018, whereas coyotes were studied from May 2018 to December 2018. Because it was important that subjects voluntarily participate in testing, we selected individuals that we perceived as bolder and/or more food motivated. Prior to the start of trials, LAS selected skunks based on 1) their 
behavioral response when she was in the building (e.g., would not hide, move away, or increase stereotypic pacing in her presence) and 2) their food motivation (e.g., showed interest in novel foods and/or "treats" presented). Based on this informal assessment of motivation to participate, LAS selected four male skunks that showed reduced fear to her presence and also showed interest in novel treats. In a similar manner, LAS selected raccoon participants that seemed less fearful to her presence in the building and were food motivated. Coyotes were selected by NWRC animal care staff based on the staff's knowledge of boldness and food motivation. Due to time constraints, investigation of any potential differences in the reversal learning abilities of bold and less bold animals did not occur.

\section{Duration Between Testing Sessions}

The median number of hours between testing sessions for all participants was 24.17 (range $=17.89-337.02)$ and the majority of testing sessions $(88 \%)$ fell between $20-30$ hours. We tested whether the duration between testing sessions influenced performance in four ways using mixed effect modeling in Program R (R Core Team 2015). Duration between testing sessions did not affect participation of test subjects (binomial GLMM: $\mathrm{p}=0.43$ ), the number of reversals each subject completed in a testing session (Poisson GLMM: 0.054, approaching but the effect size was very small: $\beta=0.004$ ), or the number of errors each participant made in either their prereversal block (Poisson GLMM: $\mathrm{p}=0.232$ ) or their first reversal (Poisson GLMM: $\mathrm{p}=0.0943$; approaching significance but the effect size is very small: $\beta=-0.002)$. In sum, there was limited variation in the time between testing sessions and we did not find strong evidence that this variation affected the performance of individuals in this experiment. 


\section{Prior experience}

The device intended for use in this experiment originally had an LCD screen that could be pushed on the left or the right to make a selection. It was our hope that subjects would be able to discriminate visual cues, and therefore we would be able to administer several types of discrimination and reversal learning tasks on the screen. We used this initial device with five raccoons and two skunks over the course of several weeks, during which time it became abundantly clear that subjects were either uninterested in interacting with the device and/or unable to understand a choice paradigm was being presented on the screen. Eventually, we decided to replace the LCD screen with two buttons in hopes that this would be more salient for the animals. We used this version of the device with three raccoons and two skunks that had 1-3 testing sessions each, and we immediately found that the button stimuli were indeed more salient to the animals. Upon this discovery, we discarded the LCD screen and ceased testing for 6 weeks so that we could rebuild our devices with the two button stimuli positioned appropriately for each species (i.e. on a vertical plane for raccoons and a low, angled plane for skunks). Subjects $(n=5)$ may have completed small number of pre-reversals (1-3) or reversals (1-10) during this pilot testing. However, because the pilot box was not working appropriately in every pilot testing session (e.g., one of the two buttons would suddenly become unresponsive), and the final protocol was not yet fully implemented, we believe it was most accurate to not include these data in our analyses.

Because skunk and raccoon testing was completed before coyote testing began, none of the coyote subjects ever had exposure to the device with the LCD screen. Initially, however, we used a device that had angled buttons similar to our skunk device, but after several testing sessions with two coyotes (Cepheus and Orion), we realized that these buttons were not 
appropriate for coyotes, and instead added pedals to the front of the device. In addition, when our single coyote participant began pushing on the pedals and releasing food from the device, he would not always remove food from the platform of the device, and this caused a backup or jamming of kibble inside of the food hopper. In response, we removed the device from his enclosure for a few days and added a tilt so that the food would fall and immediately roll forward when released from the food hopper. When we replaced the device in his enclosure and began trials, the coyote no longer showed interest in testing (i.e., for three consecutive testing sessions). At this point we ceased testing for several days and transitioned him from the typical meat-based diet onto a kibble diet. We then restarted trials with this newly angled device. At this time, the coyote performed 4 pre-reversals and 1 reversal. However, we found that the coyote was abandoning many blocks of trials during the 10 second time out, and that there was a large lag in time between choices being made. We therefore reduced the time out to 2 seconds, added the 40minute block reset, and restarted trials. Because of these differences in testing procedures, we did not include the initial 4 pre-reversals or 1 reversal in our analysis and instead only included subsequent data when the device and procedure were standardized, and participation was more consistent across testing sessions.

Once we finalized our device and protocol for all subjects, we began testing of both novel and experienced participants. We expected that prior experience may have affected subject performance in trials, especially for those animals that completed a small number of blocks with the pilot device. Thus, we categorized subjects based on their level of prior experience: group A included subjects that completed blocks during pilot testing $(\mathrm{n}=5)$, group B included subjects that had prior experience with a testing device (LCD screen, pilot device, or both) but did not 
complete blocks of trials $(\mathrm{n}=5)$, and group $\mathrm{C}$ included subjects that had no prior experience $(\mathrm{n}=$ $11)$.

\section{Habituation measures}

We implemented several measures to habituate subjects to the testing device. During skunk and raccoon trials, we programmed the device to release a reward each time a subject entered the device for up to four entries using a built-in break-beam sensor, and continued this habituation technique until they began engaging in testing (i.e., began completing blocks). We allowed up to 300 minutes per initial testing session so that the animal had time to acclimate to the device's presence, approach multiple times and receive multiple rewards. Once a subject began engaging in testing and completed multiple blocks, they no longer received multiple rewards for entering in subsequent testing sessions. Instead they only received one reward for entering the device at the beginning of subsequent testing sessions, which we used to signal the start of trials. In addition, high value food (e.g., sardines) was placed on the ground inside the device and the button stimuli were baited during a subject's first testing session but were no longer baited in subsequent testing sessions once the animal began engaging in testing. We generally began baiting the buttons lightly so that they were flavored (e.g., using water from a sardine can, cheese rubbed against button, etc.), but if this did not encourage subject participation in their first testing session, a small amount of food was added on and around the buttons in subsequent testing sessions (e.g., small amount of peanut butter or marshmallow fluff) until the animal engaged in testing. During this time subjects could push on either button and receive a reward for up to 10 pushes before the first pre-reversal test began. Subjects with prior testing experience did not receive this same habituation procedure upon the start of their trials, as they 
were already habituated to the testing device. Once an animal started testing, we reduced testing session time from 300 minutes to 30-90 minutes depending on the interest and activity level of the subject (e.g., if the animal was sleeping at the onset of trials it was provided with a longer testing session, if the animal was stress-prone it received shorter testing sessions, animals that usually demonstrated active engagement with the device received longer testing sessions, etc.).

Due to the anticipated neophobia of coyote subjects, our habituation procedure was extended for coyotes. Initially, testing sessions lasted only a few hours, during which time LAS was present at a distance observing the first two coyote subjects (Orion and Cepheus) and would remotely trigger the testing device to release food. However, after conducting several testing sessions in this manner, we decided to extend trials from dusk to dawn, during which time LAS was not present and the device was set to trigger the release of food every 40 minutes. Unlike the skunk and raccoon testing procedure, where the button stimuli were baited as little as possible, the pedal stimuli for coyotes were baited heavily with high-value food rewards. Two coyotes were always tested at a time in adjacent enclosures, and our first subjects (Orion and Cepheus) had visual access to one another. It was our hope that social facilitation might encourage coyotes to interact with the devices (Young et al. 2019), but in contrast we found that visual access to a neighbor was distracting, and so we constructed a wall between the adjacent enclosures, which was present for the remainder of testing. Similarly, to encourage participation we tried testing some coyotes $(n=2)$ both with and without their mate present in the same enclosure. Although this may have encouraged boldness and interest in the device, ultimately it did not result in participation in testing. Most of the coyotes $(n=5)$ were also transitioned from their highly preferred, meat-based diet onto a kibble diet during testing, which we hoped would increase motivation for additional high-value rewards. Ultimately, only one coyote (Orion; one of our 
initial subjects), participated in testing. In a similar study at the same NWRC facility (GilbertNorton et al. 2009), experimenters had to work hands-on with coyote subjects for 6 months in order to train them to participate in trials. Therefore, it could be that coyotes required more time to habituate, which we were unfortunately unable to provide.

\section{Results}

Participation by each subject, along with their individual traits and testing experience, is outlined in ESM Table 1. The majority of subjects (19/21) interacted with the device, by either intentionally or accidentally pushing the stimuli at least once. However, only 8/11 raccoons, 3/4 skunks and 1/6 coyotes participated in trials by completing pre-reversal and reversal blocks. One female coyote (Vela) was exhibiting increased fear of the device and was removed from trials prematurely. Similarly, one female raccoon (Luna) with prior testing experience exhibited an increase in stress during trials. In response, we shortened her testing time and allowed multiple day breaks between her testing sessions. Nevertheless, she only completed four reversals during testing. One skunk (Jupiter) only completed two reversals, and we therefore were unable to produce individual learning curves (GLMs) for Jupiter and Luna because of a lack of data. Finally, we experienced two instances of device malfunction with two raccoons: (1) the device ran out of food prematurely during the final reversal in one of Rigel's testing sessions, and (2) the device was incorrectly programmed to only deliver rewards on one side (i.e., no reversals administered) during one testing session for Pollux. We therefore removed these trials from their datasets but included data from subsequent tests. The results of individual GLMs are presented in ESM Table 3. 
ESM References:

Bartoń, K. (2018). MuMIn: Multi-Model Inference. R package version 1.42.1. https://CRAN.Rproject.org/package=MuMIn

Burnham KP, Anderson DR. (2002) A practical information-theoretic approach. Model selection and multimodel inference, 2nd ed. Springer, New York.

Gilbert-Norton LB, Shahan TA, Shivik JA. 2009. Coyotes (Canis latrans) and the matching law. Behav Processes. 82(2):178-183.

R Core Team. 2018. R: A language and environment for statistical computing. https://www.rproject.org/.

Young JK, Touzot L, Brummer SP. 2019. Persistence and conspecific observations improve problem-solving abilities of coyotes. PLoS One. 14(7):e0218778. 
ESM Table 1 Habituation, prior experience, and participation of subjects

\begin{tabular}{|c|c|c|c|c|c|c|c|c|c|}
\hline Name & ID & Species & Sex & Origin & $\begin{array}{l}\text { Ever } \\
\text { push? }\end{array}$ & $\begin{array}{l}\text { Tested with } \\
\text { mate? }\end{array}$ & $\begin{array}{l}\text { Diet } \\
\text { change? }\end{array}$ & $\begin{array}{l}\text { Prior } \\
\text { experience? }\end{array}$ & $\begin{array}{l}\text { Number of } \\
\text { reversals }\end{array}$ \\
\hline Vega & 111 & Raccoon & $\mathrm{F}$ & Wild-Caught & Yes & NA & No & B & 32 \\
\hline Pollux & 112 & Raccoon & M & Wild-Caught & Yes & NA & No & B & 32 \\
\hline Rigel & 113 & Raccoon & $\mathrm{M}$ & Wild-Caught & Yes & NA & No & $\mathrm{A}$ & 20 \\
\hline Sirius & 115 & Raccoon & M & Wild-Caught & Yes & NA & No & $\mathrm{A}$ & 23 \\
\hline Oberon & 118 & Raccoon & M & Wild-Caught & Yes & NA & No & $\mathrm{C}$ & 28 \\
\hline Astrid & 119 & Raccoon & $\mathrm{F}$ & Wild-Caught & Yes & NA & No & $\mathrm{C}$ & 13 \\
\hline Nova & 123 & Raccoon & $\mathrm{F}$ & Wild-Caught & Yes & NA & No & $\mathrm{C}$ & 0 \\
\hline Titan & 124 & Raccoon & $\mathrm{M}$ & Wild-Caught & Yes & NA & No & $\mathrm{C}$ & 0 \\
\hline Celeste & 126 & Raccoon & $\mathrm{F}$ & Wild-Caught & No & NA & No & $\mathrm{C}$ & 0 \\
\hline Castor & 128 & Raccoon & M & Wild-Caught & Yes & NA & No & $\mathrm{C}$ & 31 \\
\hline Luna & 129 & Raccoon & $\mathrm{F}$ & Wild-Caught & Yes & NA & No & $\mathrm{A}$ & 4 \\
\hline Neptune & 22 & Skunk & M & Captive-Bred & Yes & NA & No & A & 13 \\
\hline Mars & 23 & Skunk & M & Captive-Bred & Yes & NA & No & $\mathrm{C}$ & 0 \\
\hline Saturn & 26 & Skunk & M & Captive-Bred & Yes & NA & No & B & 12 \\
\hline Jupiter & 27 & Skunk & M & Captive-Bred & Yes & NA & No & $\mathrm{C}$ & 2 \\
\hline Orion & 1031 & Coyote & $\mathrm{M}$ & Wild-Caught & Yes & NA & Yes & A & 9 \\
\hline Cepheus & 1521 & Coyote & M & Captive-Bred & Yes & Yes & No & B & 0 \\
\hline Leo & 1231 & Coyote & M & Captive-Bred & Yes & Yes & Yes & $\mathrm{C}$ & 0 \\
\hline Perseus & 1151 & Coyote & M & Captive-Bred & Yes & No & No & $\mathrm{C}$ & 0 \\
\hline Draco & 1033 & Coyote & M & Wild-Caught & Yes & No & No & $\mathrm{C}$ & 0 \\
\hline Vela & 1370 & Coyote & $\mathrm{F}$ & Captive-Bred & No & No & No & $\mathrm{C}$ & 0 \\
\hline
\end{tabular}


ESM Table 2 Summary table for models evaluating the effects of reversal number, animal ID, prior experience, and species. Models were evaluated using Akaike's Information Criterion corrected for small sample sizes (AICc) and Akaike weights (Burnham and

8 Anderson, 2002). Top models were considered valid if the AICc value was the lowest of all the models, and if the Akaike weight was $9 \geq .90$. All competing models were compared to a null model (included an unspecified random intercept) and include $\mathrm{R}^{2}$ values.

10 Models were created in Program R (R Core Team 2015), and AICc values were calculated using the model.sel function in R (package:

11 MuMIn, Bartoń 2018). Top model indicated by an asterisk (*).

\begin{tabular}{lccccc}
\hline Model (Poisson GLM) & df & $\mathbf{R}^{\mathbf{2}}$ & AICc & $\Delta$ AICc & Akaike weight \\
\hline number of errors made $\sim$ reversal number + Animal ID $*$ & 13 & 0.42 & 1219.343 & 0.00 & $9.999772 \mathrm{e}-01$ \\
number of errors made $\sim$ reversal number + prior experience & 4 & 0.37 & 1240.724 & 21.38076 & $2.276231 \mathrm{e}-05$ \\
number of errors made $\sim$ reversal number + species & 4 & 0.28 & 1308.266 & 88.92234 & $4.906244 \mathrm{e}-20$ \\
number of errors made $\sim$ reversal number & 2 & 0.26 & 1316.107 & 96.76392 & $9.726841 \mathrm{e}-22$ \\
number of errors made $\sim 1$ & 1 & 0.00 & 1507.356 & 288.01328 & $2.875413 \mathrm{e}-63$ \\
\hline
\end{tabular}


ESM Table 3 Results of individual models.

\begin{tabular}{|c|c|c|c|c|c|c|c|c|c|}
\hline AID & pecies & Model (Poisson GLM) & value & lower CI & upper CI & Beta & df & z_value & $\mathbf{R 2}$ \\
\hline Orion & te & verall Reversal Nun & 31886 & -0.1 & 46163 & -0.03097 & 7 & -0.78597 & 0.015583 \\
\hline Orion & oyote & rror $\sim$ Overall Reversal $\mathrm{Nu}$ & 959258 & 0.39791 & 0.414567 & 0.010554 & 6 & 959258 & .015649 \\
\hline Orion & Coyote & Error $\sim$ Reversal Block & 2029 & -0.15497 & 0.124167 & -0.015 & 7 & 121 & 006154 \\
\hline Vega & $\mathrm{R}$ & Error & & & & & 30 & & 320028 \\
\hline Vega & Raccoon & Error $\sim$ Overall Reversal Number & 0.09 & -0.0 & 22547 & 0.283916 & 29 & 0.097243 & 370285 \\
\hline Vega & accoon & Error $\sim$ Reversal Block & 13 & -0.04227 & 0.01008 & -0.0 & 30 & 89 & 3199 \\
\hline Pollux & ccoon & Irror Overall Reversal Number & & 743 & 348 & 27 & 30 & 68 & \\
\hline Pollux & & Irror Overall Reversal Number & & & & & 29 & & \\
\hline Pollux & & Error $\sim \mathrm{I}$ & & -0 & & 17 & 30 & & 346 \\
\hline Rigel & & 然 & & & & & 10 & & \\
\hline Rigel & & Error $\sim$ Overall Reversal Number + Side & 3 & 9 & & 57 & 17 & & \\
\hline Rigel & Raccoon & Error $\sim$ Reversal Block & 54 & -0 . & 0. & -0.0 & 18 & 02 & 0.160582 \\
\hline Sirius & accoon & rror $\sim$ & 5 & 276 & -0 & 48 & 21 & 88 & \\
\hline Sirius & Raccoon & Error $\sim$ Overall Reversal Number - & 183774 & -0.10804 & 0.583329 & 0.23 & 20 & 774 & 527 \\
\hline Sirius & Raccoon & Error $\sim$ Reversal Block & 456488 & -0.05499 & 0.024486 & -0.01505 & 21 & -0.74464 & 0.15214 \\
\hline Oberon & Raccoon & Error $\sim$ Overall Reversal Number & $5 \mathrm{E}-05$ & -0.04929 & -0.0189 & -0.034 & 26 & 788 & 329 \\
\hline Oberon & Raccoon & Error $\sim$ Overall Reversal Number + Side & 0.586 & -0.17 & 0.3 & 0.067008 & 25 & 995 & 255 \\
\hline Oberon & Raccoon & Error $\sim$ Reve & & & & & 26 & & 846 \\
\hline Astrid & Raccoon & Error Overall Reversal Number & & -0.10381 & & -0.06029 & 11 & & \\
\hline Astrid & Raccoon & Error $\sim$ Overall Reversal Number & & & & & 10 & & 0.259792 \\
\hline Astrid & Raccoon & Error $\sim$ Reversal Block & & & & & 11 & & 0.308637 \\
\hline Castor & Raccoon & Error $\sim$ Overall Reversal Nu & & -0.0 & -0 & -0.0 & 29 & 88 & 0.392246 \\
\hline Castor & Raccoon & Error $\sim$ Overall Reversal Number + Side & 0.001128 & 0.18844 & 0.751212 & 0.466755 & 28 & 0.001128 & 0.456132 \\
\hline Castor & Raccoon & Error $\sim$ Reversal Block & 0.035502 & -0.05303 & -0.00206 & -0.02727 & 29 & -2.10259 & 0.230383 \\
\hline Neptune & Skunk & Error Overall Reversal Number & 0.339532 & -0.08783 & 0.029996 & -0.02864 & 11 & -0.95509 & 0.043132 \\
\hline Neptune & Skunk & Error $\sim$ Overall Reversal Number + Side & 0.290504 & -0.21013 & 0.680279 & 0.239129 & 10 & 0.290504 & 0.095148 \\
\hline
\end{tabular}




\begin{tabular}{llllllllll} 
Neptune & Skunk & Error $\sim$ Reversal Block & 0.92352 & -0.08996 & 0.099406 & 0.004609 & 11 & 0.096 & 0.007322 \\
\hline Saturn & Skunk & Error $\sim$ Overall Reversal Number & 0.001512 & -0.16548 & -0.03962 & -0.10162 & 10 & -3.17246 & 0.336939 \\
Saturn & Skunk & Error $\sim$ Overall Reversal Number + Side & 0.888964 & -0.38968 & 0.45215 & 0.029875 & 9 & 0.888964 & 0.337568 \\
Saturn & Skunk & Error $\sim$ Reversal Block & 0.296073 & -0.16581 & 0.048981 & -0.05687 & 10 & -1.04489 & 0.278899
\end{tabular}


18 ESM Video 1 Video footage from the beginning of a skunk testing session. As seen in the video,

19 skunks and raccoons were rewarded upon entering the device at the beginning of each testing

20 session. The skunk then selects the LED button to his left by pressing down on the button. This is

21 the correct response, and so a reward of dog kibble and cereal is automatically released from the

22 device. The speed of the video has been increased from its original version.

24 ESM Video 2 Video footage from a raccoon trial. The raccoon first pushes on the LED button to

25 his right side, which is the incorrect choice. In response, the lights in both LED buttons shut off

26 and the device becomes unresponsive for 10 seconds. After the 10 second time-out period has

27 passed, the raccoon returns and makes the correct selection by pushing on the button to his left

28 side. The speed of the video has been increased from its original version.

ESM Video 3 Video footage from a coyote trial. The coyote first makes a correct selection by

31 pawing on the foot pedal on his left side and therefore receives an automated reward of dog

32 kibble and sausage. After eating the reward, he then makes an incorrect selection by pawing on

33 the pedal to his right. The corresponding LED lights above the foot pedals shut off and the

34 device becomes unresponsive for two seconds. In response the coyote quickly switches back to

35 the correct choice by pawing at the pedal to his left. When the two second time-out period has

36 ended, the coyote's correct selection is registered by the device and an automated reward is

37 released by the device. 
39 ESM Video 4 Video footage from a coyote that never participated in trials. This individual 40 exemplifies the neophobic behavior displayed by most of the coyotes tested in this experiment.

41 The testing device is baited with high-value food rewards, including sausage, chicken livers, and

42 their standard, meat-based diet ("mink food"). Although the coyote is expressing interest in the

43 food by approaching the device and pawing at the ground in front of the device, he avoids

44 making contact with the testing device. This reluctance to interact with the device prevented

45 most of the coyotes tested in this study from actually participating in trials. The speed of the 46 video has been increased from its original version.

47

48

49

50 